\title{
Thomas Precession and the Bargmann-Michel-Telegdi Equation
}

\author{
Krzysztof Rębilas
}

Received: 28 September 2010 / Accepted: 18 June 2011 / Published online: 6 July 2011

(C) The Author(s) 2011. This article is published with open access at Springerlink.com

\begin{abstract}
A direct method showing the Thomas precession for an evolution of any vector quantity (a spatial part of a four-vector) is proposed. A useful application of this method is a possibility to trace correctly the presence of the Thomas precession in the Bargmann-Michel-Telegdi equation. It is pointed out that the Thomas precession is not incorporated in the kinematical term of the Bargmann-Michel-Telegdi equation, as it is commonly believed. When the Bargmann-Michel-Telegdi equation is interpreted in curved spacetimes, this term is shown to be equivalent to the affine connection term in the covariant derivative of the spin four-vector evolving in a gravitational field. It then contributes to the geodetic precession. The described problem is an interesting and unexpected example showing that approximate methods used in special relativity, in this case to identify the Thomas precession, can distort the true meaning of physical laws.
\end{abstract}

Keywords BMT equation · Spin dynamics $\cdot$ Special relativity

\section{Introduction}

In an inertial instantaneous rest frame of a moving (possibly accelerating) particle, possessing a spin $\vec{s}$, the spin dynamics is determined by the classical equation [1]:

$$
\frac{d \vec{s}}{d \tau}=\frac{g e}{2 m c} \vec{s} \times \vec{B}^{\prime},
$$

where $\vec{B}^{\prime}$ is the magnetic induction in the rest frame. To describe the dynamics of a moving spin $\vec{s}$, observed from the point of view of a laboratory system, one cannot perform simply an identification $\vec{B}^{\prime}=\vec{B}_{\|}+\gamma \vec{B}_{\perp}-\gamma \vec{\beta} \times \vec{E}$, with $\vec{B}$ being the

K. Rȩbilas ( $\varangle)$

Katedra Chemii i Fizyki, Uniwersytet Rolniczy im. Hugona Kołłątaja w Krakowie,

Al. Mickiewicza 21, 31-120 Kraków, Poland

e-mail: krzysztof.rebilas@ur.krakow.pl 
magnetic induction in the laboratory frame $(\vec{\beta}=\vec{v} / c$, where $\vec{v}$ is the instantaneous velocity of the particle). This naive procedure leads, for an electron moving in a central potential, to a spin-orbit coupling twice as large as the one required by the fine structure of hydrogen. Thomas [2] first noticed that if we connect an instantaneous inertial rest frame $R_{t}$ with the moving particle at a moment $t$ ( $t$ denotes the time measured in the laboratory frame) and next an instantaneous inertial rest frame $R_{t+d t}$ at the moment $t+d t$, and $R_{t+d t}$ is obtained from $R_{t}$ by means of a pure boost, then from the point of view of the laboratory point of view, the system $R_{t+d t}$ appears to be rotated [3-6] with respect to the system $R_{t}$ by an angle $\vec{\omega}_{T} d t$ (the Thomas precession), where:

$$
\vec{\omega}_{T}=\frac{\gamma^{2}}{\gamma+1} \frac{d \vec{\beta}}{d t} \times \vec{\beta}
$$

The reason is that the successive boosts, from the laboratory frame to $R_{t}$ and next from $R_{t}$ to $R_{t+d t}$, are in general not equivalent to a single boost but rather to a boost plus a spatial rotation. Generally then Lorentz boosts do not form a proper subgroup but, rather, generate the entire Lorentz group [7].

The Thomas precession is a purely kinematical effect but it influences the dynamics of the spin as observed in the laboratory frame [1, 2, 8-11]. The proper equation governing the movement of the spin in this system is:

$$
\left(\frac{d \vec{s}}{d t}\right)_{\text {in } L A B}=\left(\frac{d \vec{s}}{d t}\right)_{\text {in } R_{t}}+\vec{\omega}_{T} \times \vec{s}=\gamma^{-1} \frac{g e}{2 m c} \vec{s} \times \vec{B}^{\prime}+\vec{\omega}_{T} \times \vec{s},
$$

where we have made use of (1).

Another way to cope with the problem of the spin precession as seen from the point of view of the laboratory frame is a theory proposed by Bargmann, Michel and Telegdi (BMT) [12]. The spin is described by means of a four-vector $S=\left(S_{0}, \vec{S}\right)$ about which it is assumed that it coincides with $\vec{s}$ in the rest frame of the particle, i.e. has the form $(0, \vec{s})$. The law of motion for the four-spin is:

$$
\frac{d S^{\alpha}}{d \tau}=\underbrace{\frac{g e}{2 m c}\left[F^{\alpha \beta} S_{\beta}+\frac{1}{c^{2}} U^{\alpha}\left(S_{\lambda} F^{\lambda \mu} U_{\mu}\right)\right]}_{\text {torque }}-\underbrace{\frac{1}{c^{2}} U^{\alpha}\left(S_{\lambda} \frac{d U^{\lambda}}{d \tau}\right)}_{\text {kinematical term }},
$$

where $U^{\alpha}=\gamma(c, \vec{v})$ and $F^{\alpha \beta}$ is the electromagnetic field tensor. This is the most general form of the BMT equation where the acceleration of the particle $d U^{\lambda} / d \tau$ can be caused by an arbitrary (not necessarily electromagnetic) force.

It is known that (4) gives the same experimental predictions as (3). However, a theoretical problem of how the Thomas precession is incorporated into the BMT equation (4) is not correctly resolved so far. The presence of the Thomas precession in the BMT equation is generally attributed to the special form of the right side of (4) proposed by the BMT [1, 8, 11, 13]. The last term in (4) is purely kinematical and contains acceleration. What is more, this is the only term in the BMT equation in the case when the spin moves in the absence of the electromagnetic fields (formally we put $g=0$ in (4) in this case). Certainly, if no electromagnetic fields are present, the ordinary three-vector $\vec{s}$ performs only the Thomas precession (3), which 
strongly suggests that the term $\left(-1 / c^{2}\right) U^{\alpha} S_{\lambda}\left(d U^{\lambda} / d \tau\right)$ in (4) is responsible for the Thomas precession of the spin $[1,8,13]$. This however is not true. As it will be shown, even if this kinematical term is eliminated in the BMT equation (by imposing on the motion the additional constraint $\left.S_{\lambda}\left(d U^{\lambda} / d \tau\right)=0\right)$ and the BMT equation becomes $d S^{\alpha} / d \tau=0$, the three-vector $\vec{s}$ still satisfies the equation of motion of the form:

$$
\left(\frac{d \vec{s}}{d t}\right)_{\text {in } L A B}=\vec{\omega}_{T} \times \vec{s},
$$

i.e. undergoes the Thomas precession.

The difficulty with identification of the Thomas precession in the BMT equation emphasizes the fact that one can find also an attempt [11] to identify the Thomas precession with the first term of the torque in (4), i.e. with $(g e / 2 m c) F^{\alpha \beta} S_{\beta}$ (for $g=2$ ).

The aim of this paper is to develop a method to derive the Thomas precession directly for an arbitrary vector quantity, being a part of a four-vector (typically the Thomas precession is shown for systems of reference). As a direct application of this approach we point out a possibility to identify properly how the Thomas precession is incorporated in the BMT equation. We show that the commonly used approximate calculations (for $\beta \ll 1$ ), instead of exact ones, are the origin of the misleading interpretation of the BMT equation.

\section{Thomas Precession}

The Lorentz transformation for the four-spin $S=\left(S_{0}, \vec{S}\right)$ defined in the laboratory frame and the four-spin $s=\left(s_{0}, \vec{s}\right)$ (with $s_{0}=0$ ) defined in the inertial rest frame of the particle is:

$$
\begin{gathered}
s^{0}=\gamma\left(S^{0}-\vec{\beta} \cdot \vec{S}\right), \\
\vec{s}=\vec{S}+\frac{\gamma^{2}}{\gamma+1}(\vec{\beta} \cdot \vec{S}) \vec{\beta}-\gamma \vec{\beta} S^{0} .
\end{gathered}
$$

The inverse relations are:

$$
\begin{gathered}
S^{0}=\gamma\left(s^{0}+\vec{\beta} \cdot \vec{s}\right), \\
\vec{S}=\vec{s}+\frac{\gamma^{2}}{\gamma+1}(\vec{\beta} \cdot \vec{s}) \vec{\beta}+\gamma \vec{\beta} s^{0} .
\end{gathered}
$$

Because $s^{0}=0$, the above relations can be simplified. From (6) it follows that

$$
S^{0}=\vec{\beta} \cdot \vec{S}
$$

and (7) becomes:

$$
\vec{s}=\vec{S}-\frac{\gamma}{\gamma+1}(\vec{\beta} \cdot \vec{S}) \vec{\beta}
$$


However, the described below procedure requires to discern between the $\vec{\beta}$ contained in the expression (10) for $S_{0}$ and the $\vec{\beta}$ from the Lorentz transformation (7). For our purpose we will use then the transformations in the form of (6) and (7).

Let us introduce two unit vectors, one $\hat{l}$ parallel to $\vec{\beta}$ and the other $\hat{n}$ perpendicular to $\hat{l}$ such that $\vec{s}$ lies in the plane spanned by these two unit vectors. Note that $\hat{l}$ and $\hat{n}$ can serve as the base vectors in both reference frames- the laboratory and the instantaneous inertial rest system-because both are defined in an invariant way as far as these two systems are concerned. Clearly

$$
\begin{aligned}
& \hat{l}=\vec{\beta} / \beta, \\
& \hat{n} \cdot \hat{l}=0, \\
& \hat{n}^{2}=\hat{l}^{2}=1 .
\end{aligned}
$$

In effect $\vec{s}$ can be decomposed on the directions $\hat{l}$ and $\hat{n}$ :

$$
\vec{s}=s_{l} \hat{l}+s_{n} \hat{n} .
$$

On the other hand, from (9) we get:

$$
\vec{S}=\gamma s_{l} \hat{l}+s_{n} \hat{n}
$$

The components of $\vec{S}$ then are:

$$
S_{l}=\gamma s_{l}, \quad S_{n}=s_{n} .
$$

With help of (15), (13) can be now rewritten as:

$$
\vec{s}=\frac{S_{l}}{\gamma} \hat{l}+S_{n} \hat{n} .
$$

Equation (16) is a desired description of $\vec{s}$ by means of quantities used in the laboratory system. Certainly, the unit vectors $\hat{l}$ and $\hat{n}$ change in time and their change contributes to the evolution of $\vec{s}$ as seen in the laboratory frame.

Substituting $\vec{\beta}=\beta \hat{l}$ to (7), we find that (7) is precisely equivalent to (16). Therefore we conclude that (7), typically understood as the Lorentz transformation, can also be treated as a representation of $\vec{s}$ in the laboratory frame. According to this interpretation the $\vec{\beta}$ in (7) is no longer treated as a constant parameter of the Lorentz transformation but as a changing in time quantity influencing the change of $\vec{s}$ from the point of view of the laboratory frame. In the same way one can interpret (6).

Let us denote symbolically the Lorentz transformation described in (6) and (7) as $s=L S$ and perform the differentiation separating it into two terms:

$$
(\dot{s})_{\text {in LAB }}=L \dot{S}_{\left.\right|_{L(\vec{\beta})=\text { const }}}+\dot{L} S_{\mid S=\text { const }} .
$$

The dot represents the differentiation $d / d \tau$ and the condition $L(\vec{\beta})=$ const means that the $\vec{\beta}$ standing in the Lorentz transformation operator is not differentiated. Thanks to the differentiation $\dot{L}$ the whole right side of (17) actually represents the 
total rate of change of $s$ from the point of view of the laboratory frame. Introducing the reversed transformation $S=L^{-1} s$ to the first term on the right side of the above equation we obtain:

$$
L \dot{S}_{\left.\right|_{L(\vec{\beta})=\mathrm{const}}}=L\left(L^{-1} s \dot{)}_{\left.\right|_{L(\vec{\beta})=\mathrm{const}}}=L L^{-1}\left(s \dot{)}_{\left.\right|_{L(\vec{\beta})=\mathrm{const}}} \equiv(\dot{s})_{\text {in } R_{t}}\right. \text {, }\right.
$$

i.e. the ordinary transformation of the four-vector $\dot{S}$ from the laboratory frame to the instantaneous inertial rest frame of the particle $R_{t}$.

In turn, the second term on the right side of (17) can be rewritten as:

$$
\dot{L} S=\frac{L(t+d t)-L(t)}{d t} L(t)^{-1} s=\frac{L(t+d t) L^{-1}(t)-1}{d t} s,
$$

where $L(t) \equiv L(\vec{\beta}(t))$ and $L(t+d t) \equiv L(\vec{\beta}(t+d t))$. Because in general $\vec{\beta}(t)$ and $\vec{\beta}(t+d t)$ need not be collinear, the sequence of the pure boosts $L(t+d t) L^{-1}(t)$ is known [1] to contain the Thomas precession. To calculate explicitly the term $\dot{L} S_{\mid S=\text { const }}$ we notice that:

$$
\dot{L} S_{\mid S=\text { const }}=\left(L S \dot{)}_{\mid S=\text { const }}=\left(s \dot{~}_{\left.\right|_{S=\text { const }}}\right.\right.
$$

and for the spatial part of $\dot{L} S_{\left.\right|_{S=\text { const }}}$, from (7), we get:

$$
\begin{aligned}
\dot{\dot{L}} S_{\mid S=\text { const }}=(\vec{s})_{\mid S=\text { const }}= & \left(\frac{\gamma^{2}}{\gamma+1}\right)(\vec{\beta} \cdot \vec{S}) \vec{\beta}+\frac{\gamma^{2}}{\gamma+1}(\dot{\vec{\beta}} \cdot \vec{S}) \vec{\beta}+\frac{\gamma^{2}}{\gamma+1}(\vec{\beta} \cdot \vec{S}) \dot{\vec{\beta}} \\
& -\dot{\gamma} \vec{\beta} S^{0}-\gamma \dot{\vec{\beta}} S^{0} .
\end{aligned}
$$

Using the relations:

$$
\dot{\gamma}=\gamma^{3}(\vec{\beta} \cdot \dot{\vec{\beta}}), \quad\left(\frac{\gamma^{2}}{\gamma+1}\right)=\frac{(\gamma+2) \gamma^{4}(\vec{\beta} \cdot \dot{\vec{\beta}})}{(\gamma+1)^{2}}
$$

one can perform the differentiation in (21) (we omit here the explicit result). Next we express $S$ by means of $s$ using (8) and (9). After elementary calculations we find that the terms proportional to $(\vec{\beta} \cdot \dot{\vec{\beta}})(\vec{\beta} \cdot \vec{s}) \vec{\beta}$ cancel out, and from (21) we get:

$$
\dot{\dot{L}} S_{\mid S=\text { const }}=\frac{\gamma^{2}}{\gamma+1}[\vec{s} \times(\vec{\beta} \times \dot{\vec{\beta}})]-\gamma s^{0} \dot{\vec{\beta}}-\frac{\gamma^{3}}{\gamma+1}(\vec{\beta} \cdot \dot{\vec{\beta}}) s^{0} \vec{\beta} .
$$

Because $s^{0}=0$ :

$$
\overrightarrow{\dot{L} S_{\mid S=\text { const }}}=\frac{\gamma^{2}}{\gamma+1}[\vec{s} \times(\vec{\beta} \times \dot{\vec{\beta}})]
$$

or, recalling the definition (2):

$$
\overrightarrow{\dot{L} S_{\mid S=\text { const }}}=\gamma \vec{\omega}_{T} \times \vec{s} .
$$


Inserting our results (18) and (25) into (17) we arrive at:

$$
(\dot{\vec{s}})_{\text {in LAB }}=(\dot{\vec{s}})_{\text {in } R_{t}}+\gamma \vec{\omega}_{T} \times \vec{s},
$$

which is the result (3) that is typically derived indirectly, i.e. on the basis of the behavior of systems of reference.

\section{Thomas Precession and the BMT Equation}

In the previous section $\vec{s}$ is treated formally as an element of the BMT theory. It is the spatial part of the four-vector $S$ (written in the rest frame) satisfying the condition $s^{0}=0$. Remaining within the BMT theory, to introduce dynamics to the purely kinematical relation (26), we refer to the BMT equation (4). Certainly, in the context of (26), we need to know the BMT equation only in the rest frame $R_{t}$ where it reduces (its spatial part) to (1). Thus (26) becomes:

$$
(\dot{\vec{s}})_{\text {in } \mathrm{LAB}}=\underbrace{\frac{g e}{2 m c} \vec{s} \times \vec{B}^{\prime}}_{\text {torque }}+\underbrace{\gamma \vec{\omega}_{T} \times \vec{s}}_{\text {kinematical term }} .
$$

This equation and the original one (4) are completely equivalent; they both determine behavior of the spin from the point of view of the laboratory frame but in (27) it is expressed in terms the new variables, $\vec{s}$ instead of $S$. Structurally (27) resembles (4); there is a term representing the torque and the kinematical term in the both equations. This suggests that the term $\gamma \vec{\omega}_{T} \times \vec{s}$ exhibiting the Thomas precession in (27) emerges from the kinematical term $\left(-1 / c^{2}\right) U^{\alpha}\left(S_{\lambda} \dot{U}^{\lambda}\right)$ present in (4). This however is not true.

To make it evident, consider the special case with $g=0$ and the additional constraint $S_{\lambda} \dot{U}^{\lambda}=0$ imposed on the particle motion. Then the BMT equation (4) becomes $\dot{S}=0$ and does not contain the kinematical term that is commonly regarded as responsible for the Thomas precession. How is in this case the equivalent to the BMT equation (27)? As $\dot{S}=0$, from (17) we get $(\dot{s})_{\text {in LAB }}=\dot{L} S_{\left.\right|_{S=\text { const }}}$ and using (25) we get:

$$
(\dot{\vec{s}})_{\text {in LAB }}=\gamma \vec{\omega}_{T} \times \vec{s} .
$$

This is the spatial part of the simplified BMT equation, $\dot{S}=0$, but expressed in terms of $\vec{s}$. As can be seen, despite the lack of the term $\left(-1 / c^{2}\right) U^{\alpha}\left(S_{\lambda} \dot{U}^{\lambda}\right), \vec{s}$ still undergoes the Thomas precession. The term $\left(-1 / c^{2}\right) U^{\alpha}\left(S_{\lambda} \dot{U}^{\lambda}\right)$ in the BMT equation cannot then be understood as responsible for the Thomas precession.

In general, if we trace the derivation of (26) starting from the decomposition of the differentiation (17), we see that the term $\gamma \vec{\omega}_{T} \times \vec{s}$ in (27) comes solely from $\dot{L} S$ (see (25)) where there is no space for any term from the BMT equation (4). Both the torque and the kinematical term standing on the right side of the BMT equation determine $\dot{S}$ so that they are incorporated into the term $L \dot{S}$. As it has been shown, the term $L \dot{S}$ does not yield $\gamma \vec{\omega}_{T} \times \vec{s}$ but rather $(\dot{\vec{S}})_{\text {in } R_{t}}$ (see (18)) that has nothing in common with the Thomas precession. In effect, the whole right side of the BMT 
equation determining the value of $\dot{S}$ is completely irrelevant for the Thomas precession, which is additionally shown explicitly in (45). It appears that important is only the Lorentz transformation $L$ between $S$ and $s$ which introduces to the rate of change of $\vec{s}$ the term $\dot{L} S$ (17) being directly equivalent to the Thomas precession.

An interesting additional remark can be made as concerns the relation of the BMT equation to general relativity. The foundations of the spin dynamics in gravitational fields were laid by Papapetrou [14] and Schiff [15] and recently an original approach was presented by Obukhov, Silenko and Teryaev [16]. The BMT equation is commonly attributed to the case of electromagnetic fields accelerating particles and causing a torque on the spin. We want to show that the BMT equation can be also applied to the gravitational fields within the framework of general relativity. The BMT equation for a particle accelerated solely by gravitational forces is:

$$
\frac{d S^{\alpha}}{d \tau}=-\frac{1}{c^{2}} U^{\alpha}\left(S^{\lambda} \frac{d U_{\lambda}}{d \tau}\right) .
$$

In [16] it is suggested that an equation of the form $d S^{\alpha} / d \tau=\Phi_{\beta}^{\alpha} S^{\beta}$ can be interpreted as a correct equation of motion in curved spacetimes, i.e. as precisely equivalent to $D S^{\alpha} / d \tau=0$. It follows then that in the BMT equation (29) one should identify:

$$
\frac{1}{c^{2}} U^{\alpha}\left(S^{\lambda} \frac{d U_{\lambda}}{d \tau}\right)=U^{\nu} \Gamma_{\nu \beta}^{\alpha} S^{\beta}
$$

Let us prove that (30) is identically satisfied. Inserting $U^{\nu} U_{\beta} \Gamma_{\nu \lambda}^{\beta}$ for $d U_{\lambda} / d \tau$ and using coordinates $\xi^{\mu}$ of a local frame, the left side of this equation reads:

$$
\begin{aligned}
\frac{1}{c^{2}} U^{\alpha} S^{\lambda} U^{\nu} U_{\beta} \Gamma_{\nu \lambda}^{\beta} & =\frac{1}{c^{2}} U^{\alpha} S^{\lambda} U^{\nu} U_{\beta} \frac{\partial x^{\beta}}{\partial \xi^{i}} \frac{\partial^{2} \xi^{i}}{\partial x^{\nu} \partial x^{\lambda}} \\
& =\frac{1}{c^{2}} \frac{d x^{\alpha}}{d \tau} S^{\lambda} U^{\nu} \frac{d x_{\beta}}{d \tau} \frac{d x^{\beta}}{d \tau} \frac{d \tau}{d \xi^{i}} \frac{\partial^{2} \xi^{i}}{\partial x^{\nu} \partial x^{\lambda}} \\
& =S^{\lambda} U^{\nu} \frac{\partial x^{\alpha}}{\partial \xi^{i}} \frac{\partial^{2} \xi^{i}}{\partial x^{\nu} \partial x^{\lambda}} \\
& =S^{\lambda} U^{\nu} \Gamma_{\nu \lambda}^{\alpha},
\end{aligned}
$$

which is precisely the right side of (30). The kinematical term of the BMT appears to be equivalent to the affine connection term in the covariant derivative $D S^{\alpha} / d \tau$ for the spin evolving in a gravitational field. It is then responsible for the geodetic precession rather than for the Thomas precession.

\section{Remarks and Conclusions}

The decomposition of the differentiation made according to (17) has appeared to be useful in demonstrating the Thomas precession for the spin vector $\vec{s}$ and identifying this effect in the BMT equation. It is worth explaining the origin of the common mistake of ascribing the Thomas precession to the kinematical term in the BMT 
equation. In Appendix we give a detailed discussion aimed to compare directly the approximate approach to the BMT equation accessible in the literature and the exact one offered in this paper. It is shown that the approximate method leads, within its limits, to a qualitatively correct result but the approximations used entail incorrect identification of the Thomas precession in the BMT equation. This is an extraordinary and unexpected situation when approximations made in special relativity distorts the theoretical meaning of the physical law.

Acknowledgements I wish to thank P. Prawda for his assistance in analyzing the nuances of the BMT theory.

Open Access This article is distributed under the terms of the Creative Commons Attribution Noncommercial License which permits any noncommercial use, distribution, and reproduction in any medium, provided the original author(s) and source are credited.

\section{Appendix: Two Ways of Showing the Thomas Precession in the BMT Equation}

\section{A.1 Approximate Method}

The most clear presentation (using approximate calculations) of how the Thomas precession is incorporated into the BMT equation can be found in [8]. The dynamics of the spin for a scalar field or for the electromagnetic field formally eliminated $(g=0)$ is described by the BMT equation in the form:

$$
\frac{d S^{\alpha}}{d \tau}=-\frac{1}{c^{2}} U^{\alpha}\left(S_{\lambda} \frac{d U^{\lambda}}{d \tau}\right)
$$

An approximate version of this equation, based on the simplification $\beta \ll 1$, is:

$$
d \vec{S} / d t \simeq \vec{\beta} \vec{S} \cdot(d \vec{\beta} / d t)
$$

We can add to the both sides of this equation the term $-d / d t\left(\frac{1}{2} \vec{\beta} \vec{\beta} \cdot \vec{S}\right)$ and obtain:

$$
\begin{aligned}
d \vec{S} / d t-d / d t\left(\frac{1}{2} \vec{\beta} \vec{\beta} \cdot \vec{S}\right) & \simeq \vec{\beta} \vec{S} \cdot(d \vec{\beta} / d t)-d / d t\left(\frac{1}{2} \vec{\beta} \vec{\beta} \cdot \vec{S}\right) \\
& \simeq \frac{1}{2}[(d \vec{\beta} / d t) \times \vec{\beta}] \times \vec{S}
\end{aligned}
$$

The left side is the rate of change of the vector:

$$
\vec{S}=\vec{S}-\frac{1}{2} \vec{\beta} \vec{\beta} \cdot \vec{S}
$$

[note that this is a simplified version of (11), as expected], so that (34) is the evolution of $\vec{s}$ in the laboratory frame:

$$
(d \vec{s} / d t)_{\text {in } L A B} \simeq \frac{1}{2}[(d \vec{\beta} / d t) \times \vec{\beta}] \times \vec{S} \simeq \frac{1}{2}[(d \vec{\beta} / d t) \times \vec{\beta}] \times \vec{s} \simeq \vec{\omega}_{T} \times \vec{s},
$$


exhibiting the Thomas precession. According to the derivation of (36), the kinematical term $\vec{\beta} \vec{S} \cdot(d \vec{\beta} / d t)$ partially (because a half of it is canceled out by the respective term from the expression $-d / d t\left(\frac{1}{2} \vec{\beta} \vec{\beta} \cdot \vec{S}\right)$, see (34)) contributes to the term representing the Thomas precession.

\section{A.2 Exact Method}

Analogous but performed without any approximations transformations lead to a completely different conclusion as concerns the participation of the kinematical term of the BMT equation in the Thomas precession. The spatial part of (32) precisely is:

$$
\dot{\vec{S}}=\gamma^{2} \vec{\beta} \vec{S} \cdot \dot{\vec{\beta}}
$$

To introduce $\vec{s}$ we add to the both sides the term $-\left(\frac{\gamma}{\gamma+1}(\vec{\beta} \cdot \vec{S}) \vec{\beta}\right)$ (see (11)):

$$
\dot{\vec{S}}-\left(\frac{\gamma}{\gamma+1}(\vec{\beta} \cdot \vec{S}) \vec{\beta}\right)=\gamma^{2} \vec{\beta} \vec{S} \cdot \dot{\vec{\beta}}-\left(\frac{\gamma}{\gamma+1}(\vec{\beta} \cdot \vec{S}) \vec{\beta}\right)
$$

and the left side represents $(\dot{\vec{s}})_{\text {in LAB }}$ (the $\vec{\beta}$ is treated as a quantity changing in time):

$$
(\dot{\vec{S}})_{\text {in LAB }}=\gamma^{2} \vec{\beta} \vec{S} \cdot \dot{\vec{\beta}}-\left(\frac{\gamma}{\gamma+1}(\vec{\beta} \cdot \vec{S}) \vec{\beta}\right)
$$

Deriving the difference $\vec{s}-\vec{S}$ from (7) and (10), we have identity:

$$
-\left(\frac{\gamma}{\gamma+1}(\vec{\beta} \cdot \vec{S}) \vec{\beta}\right)=\left(\frac{\gamma^{2}}{\gamma+1}(\vec{\beta} \cdot \vec{S}) \vec{\beta}-\gamma \vec{\beta} S^{0}\right)
$$

The differentiation can be made in two steps:

$$
\begin{aligned}
& \left(\frac{\gamma^{2}}{\gamma+1}(\vec{\beta} \cdot \vec{S}) \vec{\beta}-\gamma \vec{\beta} S^{0}\right) \\
& \quad=\left.\left(\frac{\gamma^{2}}{\gamma+1}(\vec{\beta} \cdot \vec{S}) \vec{\beta}-\gamma \vec{\beta} S^{0}\right)\right|_{L(\vec{\beta})=\mathrm{const}}+\left.\left(\frac{\gamma^{2}}{\gamma+1}(\vec{\beta} \cdot \vec{S}) \vec{\beta}-\gamma \vec{\beta} S^{0}\right)\right|_{S=\text { const }}
\end{aligned}
$$

On the basis of (7), we have:

$$
\left.\left(\frac{\gamma^{2}}{\gamma+1}(\vec{\beta} \cdot \vec{S}) \vec{\beta}-\gamma \vec{\beta} S^{0}\right)\right|_{L(\vec{\beta})=\mathrm{const}}=(\dot{\overrightarrow{\vec{S}}})_{\mathrm{in} R_{t}}-(\dot{\vec{S}})_{\mathrm{in} \mathrm{LAB}}=-(\dot{\vec{S}})_{\text {in LAB }}
$$

where the last equality follows from the fact that $g=0$. In turn, again thanks to (7), we get:

$$
\left.\left(\frac{\gamma^{2}}{\gamma+1}(\vec{\beta} \cdot \vec{S}) \vec{\beta}-\gamma \vec{\beta} S^{0}\right)\right|_{S=\mathrm{const}}=\overrightarrow{\dot{L} S_{\mid S=\mathrm{const}}}=(\vec{s})_{\left.\right|_{S=\mathrm{const}}}=\gamma \vec{\omega}_{T} \times \vec{s},
$$


where the last equality has already been proved earlier (26). Finally then the last term in (39) is

$$
-\left(\frac{\gamma}{\gamma+1}(\vec{\beta} \cdot \vec{S}) \vec{\beta}\right)=-(\dot{\vec{S}})_{\text {in LAB }}+\gamma \vec{\omega}_{T} \times \vec{s}
$$

and (39) becomes:

$$
(\dot{\vec{s}})_{\text {in LAB }}=\gamma^{2} \vec{\beta} \vec{S} \cdot \dot{\vec{\beta}}-(\dot{\vec{S}})_{\text {in LAB }}+\gamma \vec{\omega}_{T} \times \vec{s}=\gamma \vec{\omega}_{T} \times \vec{s} .
$$

The last equality follows from (37) that makes the kinematical term $\gamma^{2} \vec{\beta} \vec{S} \cdot \dot{\vec{\beta}}$ be canceled out by the term- $-(\dot{\vec{S}})_{\text {in LAB }}$. Although (45) is consistent with the approximate result (36), here the term representing the Thomas precession does not emerge from the kinematical term $\gamma^{2} \vec{\beta} \vec{S} \cdot \dot{\vec{\beta}}$. The Thomas rotation comes solely from the terms necessary to perform the transformation from $\vec{S}$ to $\vec{s}$. This fact is veiled in the approximate approach where the kinematical term is canceled out only partially and seems to contribute to the Thomas precession.

\section{References}

1. Jackson, J.D.: Classical Electrodynamics. Wiley, New York (1962), Sects. 11.8 and 11.11

2. Thomas, L.H.: The kinematics of an electron with an axis. Philos. Mag. 3, 1-22 (1927)

3. Goedecke, G.H.: Geometry of the Thomas precession. Am. J. Phys. 46, 1055-1056 (1978)

4. Rowe, E.G.P.: Rest frames for a point particle in special relativity. Am. J. Phys. 64, 1184-1196 (1996)

5. Hamilton, J.D.: Relativistic precession. Am. J. Phys. 64, 1197-1201 (1996)

6. Farach, H.A., Aharonov, Y., Poole, C.P., Jr., Zanette, S.I.: Application of the nonlinear vector product to Lorentz transformations. Am. J. Phys. 47, 247-249 (1979)

7. Misner, C.W., Thorne, K.S., Wheeler, J.A.: Gravitation. Freeman, San Francisco (1973), p. 1146

8. Schwinger, J.: Spin precession—a dynamical discussion. Am. J. Phys. 42, 510-513 (1974)

9. Strandberg, M.W.P.: Special relativity completed: the source of some $2 \mathrm{~s}$ in the magnitude of physical phenomena. Am. J. Phys. 54, 321-331 (1986)

10. Muller, R.A.: Thomas precession: where is the torque? Am. J. Phys. 60, 313-317 (1992)

11. Munoz, G.: Spin-orbit interaction and the Thomas precession: a comment on the lab frame point of view. Am. J. Phys. 69, 554-556 (2001)

12. Bargmann, V., Michel, L., Telegdi, V.L.: Precession of the polarization of particles moving in a homogeneous electromagnetic field. Phys. Rev. Lett. 2, 435-436 (1959)

13. Panofsky, W.K., Phillips, M.: Classical Electricity and Magnetism. Addison-Wesley, Reading (1962), Sect. $23-5$

14. Papapetrou, A.: Proc. R. Soc. Lond. Ser. A, Math. Phys. Sci. 209(1097), 248-258 (1951)

15. Schiff, L.I.: Phys. Rev. Lett. 4, 215 (1960)

16. Obukhov, Y.N., Silenko, A.J., Teryaev, O.V.: Phys. Rev. D 80, 064044 (2009) 